\title{
Synovial fluid detection in intra-articular injections using a bioimpedance probe (BIP) needle-a clinical study
}

\section{Halonen, Sanna}

2017-06

Halonen , S , Kankaanpää , E , Kari , J , Parmanne , P , Relas , H , Kronstrom , K, Luosujärvi , R \& Peltomaa , R 2017 , ' Synovial fluid detection in intra-articular injections using a bioimpedance probe (BIP) needle-a clinical study ' , Clinical Rheumatology , vol. 36 , no. 6 , pp. 1349-1355 . https://doi.org/10.1007/s10067-016-3210-4

http://hdl.handle.net/10138/237059

https://doi.org/10.1007/s10067-016-3210-4

unspecified

publishedVersion

Downloaded from Helda, University of Helsinki institutional repository.

This is an electronic reprint of the original article.

This reprint may differ from the original in pagination and typographic detail.

Please cite the original version. 


\title{
Synovial fluid detection in intra-articular injections using a bioimpedance probe (BIP) needle—a clinical study
}

\author{
Sanna Halonen ${ }^{1}$ E Eeva Kankaanpää ${ }^{2}$ Juho Kari ${ }^{1} \cdot$ Pinja Parmanne $^{2} \cdot$ Heikki Relas $^{2}$ • \\ Kai Kronström ${ }^{1}$ • Riitta Luosujärvi ${ }^{2}$ - Ritva Peltomaa ${ }^{2,3}$
}

Received: 25 January 2016 /Revised: 29 January 2016 / Accepted: 2 February 2016 /Published online: 12 February 2016

(C) International League of Associations for Rheumatology (ILAR) 2016

\begin{abstract}
Intra-articular glucocorticoid injections are the recommended treatment for active arthritis, but accurate positioning of the needle may be challenging. Inexperienced physicians might decide not to inject because an unsuccessful injection impairs clinical outcome and may lead to complications; however, choosing not to inject may impair or delay the best possible treatment. Here, we address this problem by introducing a novel Bioimpedance Probe (BIP) Needleguidance method that was tested in a clinical study. The BIP Needle was utilized for detection of synovial fluid. It measures real-time bioimpedance spectra and identifies when the needle tip is in contact with the synovial fluid. Injections into 80 joints with active arthritis were performed by an experienced rheumatologist using the BIP Needle. The location of the BIP Needle was ensured by aspiration of synovial fluid, absence of resistance during injection, and/or using real-time ultrasound imaging. Sensitivity and specificity of the device for synovial fluid detection were $86 \%$ (CI 75-93\%) and $85 \%$ (CI 74$92 \%)$, respectively. The BIP Needles showed high spatial resolution and differentiated the synovial fluid from the surrounding tissues. However, lack of synovial fluid, anatomic variability, and intra-articular structures challenged the technology. The BIP Needles provided adequate results in intra-
\end{abstract}

Ritva Peltomaa

ritva.peltomaa@hus.fi

R\&D Department, Injeq Ltd., Tampere, Finland

2 Department of Medicine, Division of Rheumatology, Helsinki University Central Hospital and University of Helsinki, Helsinki, Finland

3 Helsinki University Central Hospital, Helsinki Triangle Hospital, Haartmaninkatu 4, PO Box 372, FI-00029 HUS Helsinki, Finland articular injections. Performance of the device was good even in small joints, which may be the most difficult for inexperienced physicians. Further performance improvement can be expected when more data is collected for mathematical models. Overall, this novel method showed potential to be used in real-time needle guidance.

Keywords Arthritis · Bioimpedance · Corticosteroids · Intra-articular injection $\cdot$ Needle guidance

\section{Introduction}

The main outcome target in the treatment of rheumatic arthritis (RA) is full remission. Early control of inflammation with disease-modifying antirheumatic drugs (DMARDs) and systemic or intra-articular glucocorticoids (GCs) has been shown to be effective in reaching remission [1]. GC injections are the traditional treatment and are considered a safe and effective method in treating RA [2, 3]. For example in gout, occurrence of bacterial arthritis was only about 1 in 1000 punctures [4]. Active treatment of arthritis with GC injections has been shown to retard radiological progression [5] and increase remission rates [6].

Systemic treatment is often started using synthetic DMARDs (sDMARDs) and active inflammation is kept in control with intra-articular GC injections. During the past few years, biological DMARDs (bDMARDs) have been studied intensively and have been found to be effective in patients who do not respond to sDMARDs. However, in most cases, sDMARDs are as effective as bDMARDs but significantly less costly [7, 8]. Regardless of the type of DMARD used, intra-articular GC injections are recommended as part of the treatment of active arthritis $[9,10]$. They should not be dismissed, because 
neglecting use of GC injections may impair remission rates and lower quality of life [6].

Despite the recommendations, patients may not receive GCs when needed. One problem with using GC intra-articular injections is difficulty in determining the exact positioning of the needle. The success rate in needle positioning for injections guided only by clinical palpation is around 30-80\% [11, 12]. Real-time needle-guidance methods have been developed for more accurate needle location. For example, use of ultrasonography significantly increased the accuracy, from 32 to $97 \%$, in the study of Balint et al. [11]. A newer method for needle guidance is utilization of bioimpedance [13].

We report our results from a clinical study of a novel bioimpedance-based needle-guidance method, which we term Bioimpedance Probe (BIP) Needle. This method provides realtime feedback to the physician during the injection and alerts when the needle is in contact with synovial fluid. The aim is that an experienced physician can increase the accuracy of their needle positioning, while an inexperienced physician is assisted with the procedure and therefore encouraged to use the injections. This is the first time, to our knowledge, that a bioimpedance-based, tissue-sensing technology has been utilized in intra-articular GC injections in patients with RA and other arthritides. The study assessed the needle-guidance method for detection of synovial fluid.

\section{Materials and methods}

\section{Study design}

This clinical study was authorized by the Ethics Committee of the Department of Medicine, Helsinki University Central Hospital (79/13/03/01/2014). The procedures followed were in accordance with the ethical standards of the responsible committee on human experimentation and with the Helsinki Declaration of 1975, as revised in 1983. All participants provided written informed consent.

The study was performed in the rheumatology outpatient clinic of Helsinki University Central Hospital, which is Finland's largest such clinic. The patients were treated according to normal treatment strategies. The intra-articular injection procedure did not differ from that used in normal practice, except for the use of the BIP Needle and a bioimpedance analyzer, which detected synovial fluid during the injection.

Before the injection, the target joint was clinically evaluated, including ultrasonography examination, and the most suitable injection site was identified. An experienced rheumatologist performed the injection and evaluated the performance of the measurement device. Small joints were injected with $24 \mathrm{G}$ needles and larger joints with $22 \mathrm{G}$ needles. When the target was reached, correct needle placement was confirmed by aspiration of synovial fluid, ultrasound imaging, and/or absence of resistance when injecting GC. Injections were excluded from the statistical analysis $(n=8)$ if there was needle placement uncertainty, clear user error, a connection error in the cable, or a problem with the power supply. Regardless of the operation of the measurement device, the GC injection was given when clinically indicated.

The final numbers of injections included in the statistical analysis were 15 and 57 with the $22 \mathrm{G}$ and $24 \mathrm{G}$ needles, respectively, giving a total of 72 injections. Three injections did not reach the synovial cavity, as verified by ultrasound imaging, but were included in the analysis.

The rheumatologist filled in a case report form immediately after each treatment and evaluated the performance of the device. The form included both a written description and a table for true-/false-positive and true-/false-negative detection of the target. The injection was treated as a whole, meaning that even a single false event was sufficient to determine the whole detection false, even though most of the time the device functioned correctly.

\section{Patients}

All suitable adult patients that came to the clinic for GC injection were given the opportunity to join the study. Thus, the test subjects were randomly chosen and represented the usual distribution of patients in this rheumatology department.

The mean age of the patients $(N=51)$ was 53 years (range 19-84 years), and $74 \%$ were female. The diagnoses and
Table 1 Baseline characteristics (80 injections)

\begin{tabular}{llll}
\hline Diagnosis & \multicolumn{3}{c}{ Disease duration (year) } \\
\hline Rheumatoid arthritis (RF+) & 44 & $0-1$ & 10 \\
Rheumatoid arthritis (RF-) & 10 & $2-5$ & 22 \\
Oligoarthritis seronegative & 10 & $6-10$ & 14 \\
Chronic juvenile arthritis & 5 & $11-$ & 34 \\
Others: ankylosing spondylitis (4), Sjögren syndrome & 11 & &
\end{tabular}

(2), reactive arthritis (2), and psoriatic arthritis (3) 
disease durations are presented in Table 1. Each injection was studied individually regardless of the other possible injections listed, so that the data are based on the number of injections rather than the number of patients (some patients received multiple injections). Thus, the statistics of the test subject represent the statistics of the studied injections.

The distribution of the joints injected is shown in Table 2. Knees were excluded from the study because they were considered easy to inject and, therefore, were meaningless for assessing later practical use of BIP Needle. The total number of injected joints was 80 joints in 51 patients; thus, the mean number of injected joints was 1.57 per patient.

\section{Measurement system}

Measurements were performed using the BIP Needle (see Fig. 1) connected to the measurement device and tissueclassification software [14]. The BIP Needle is a standard $22 \mathrm{G}$ or $24 \mathrm{G}$ hypodermic needle, but inside the needle is a removable BIP stylet. During needle positioning, the BIP is kept inside the needle, enabling measurement from the needle tip. When the target is reached, the BIP is removed and the GC injected.

The BIP Needle is connected to a measurement device and a laptop containing tissue-classification software. The software parameters are optimized for each needle type, that is, there are specific mathematical models for the $22 \mathrm{G}$ and $24 \mathrm{G}$ needles. The bioimpedance spectrum is measured at multiple frequencies in the kilohertz range. Based on the measured impedance and phase angle values, the software classifies the tissue in which the needle tip is located, using the mathematical model. When the target tissue is detected, the device beeps, providing a clear feedback to the physician during the injection.

The original mathematical model was based on animal studies with pigs, and then the parameters were fine-tuned during an exploratory phase clinical study [14, 15]. In that study, two target classes for synovial fluid were created: "Pure" and "Complex." The Pure class represents the case when the needle tip is in good contact with the pure, well- conducting synovial fluid; such data are obtained from, for example, knees with effusion. In the exploratory phase, it was noted that in small and rheumatic joints with small amounts of synovial fluid, the bioimpedance spectra differed from the pure synovial fluid; therefore, a new class labeled Complex was determined. Class Complex is less certain detection.

\section{Statistical analysis}

Sensitivity, specificity, positive predictive power, and negative predictive power were calculated from the results. Detection of Pure and Complex cases were treated in the same manner, e.g., a case was considered true positive if the device returned either a Pure or Complex result when the synovial fluid was reached, whereas if the device returned a Pure or Complex result when the needle was not in the synovial fluid, the whole injection was considered false positive.

Confidence interval was calculated for $95 \%$ certainty intervals using the Clopper-Pearson method and binomial distribution. Two different needle types were used and different joints injected, but statistically it is relevant to consider only the pooled results.

\section{Results}

The device functioned correctly in $74 \%(53 / 72)$ of injections (Table 3). It detected either Pure, Complex, or Complex plus Pure synovial fluid in successful injections. In three injections, the needle did not reach the synovial cavity and the device did not provide any detection. Most of the detections were Complex detections. Pure detections were obtained in $29 \%$ (21/72) of correct detections, but during these injections, Complex detections also occurred frequently. The BIP Needle was sensitive to spatial placement, and even small movements affected the classification results. Normal practice of the clinic was followed, and no bacterial contamination after injection or any other safety issues or risks appeared during this clinical study.
Table 2 Injected joints $(N=80)$ and correct functioning of the device in different joints

\begin{tabular}{llll}
\hline Target joint & Injected & Included to analysis & Correct functioning \\
\hline Wrist & 26 & 24 & 18 \\
Ankle & 14 & 12 & 8 \\
MCP joint & 12 & 12 & 9 \\
MTP joint & 11 & 9 & 9 \\
Hip & 6 & 6 & 4 \\
Others: finger (4), shoulder (4), and elbow (3) & 11 & 9 & 5 \\
\hline
\end{tabular}

$M C P$ metacarpophalangeal, MTP metatarsophalangeal 


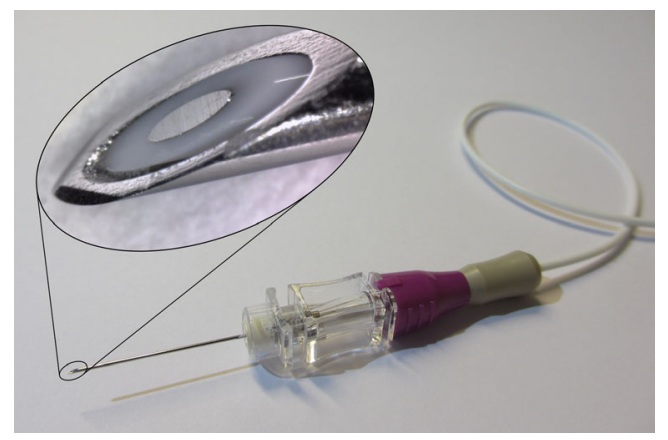

Fig. $124 \mathrm{G}$ injection needle with bioimpedance probe (BIP) stylet connected to the measurement device. Miniature electrode configuration enables highly local and spatially precise measurement from the very tip of the needle. Reproduced from [14]

Typically, large joints with excess synovial fluid provided Pure detections, while Complex detections occurred in small joints. Figure 2 presents three example cases of injections, into small, medium, and large joints. The figure shows the proportion of detections within 1-s time intervals as a bar plot, and the median filtered impedance value as a line plot. The impedance spectra are needed for the sophisticated mathematical model, but single-frequency impedance provides a rough idea of the properties of the tissue. In each case, the impedance is high at the beginning of the injection because the needle tip is in poorly conducting tissue. As the needle proceeds through the tissue, the impedance value varies according to the tissue type and tissue structures. Motion artifacts also cause fluctuations in the values. Finally, when the needle is in the synovial fluid, the impedance value is low and the device provides the detection feedback.

Movement inside the synovial cavity also affected the impedance, probably due to the inner structures of the joint. For example, in wrist injection (Fig. 2), the synovial cavity was attained quickly, contact was good, and detection result was Pure. However, Complex detection then occurred, probably as a result of needle movement.
When the needle position was changed further, Pure detection was achieved again. Hip injection took a longer time to complete than other injections, but when the synovial cavity was reached, the detection was clear and Pure was detected immediately. In metacarpophalangeal (MCP) injection, only Complex detections were achieved, which was typical of small-joint injections. In these joints, the cavity size and amount of synovial fluid are small, which both influence measurement results.

The classification model for $24 \mathrm{G}$ needles detected synovial fluid in 50 injections when the needle was in the joint cavity. In 10 injections, the device provided false-positive detection; the target joints in these cases were five wrists, three ankles and one elbow, and one MCP. In many cases, these false detections were very short and occurred with rapid needle movements.

All detections except one were correct with the $22 \mathrm{G}$ needles. In four injections (one elbow joint, one ankle joint, and two hip joints), the needle tip was in the joint cavity but the device did not beep. Correct functioning of the device in different joints is listed specifically in Table 2.

When the results from both needles were combined together, the sensitivity was found to be $86 \%$ and the specificity $85 \%$. These and other statistics with confidence intervals are shown in Table 4. The calculated statistical performance values are on the same level with each other. According to the confidence intervals, even the worst performance result was on the level of $75 \%$.

\section{Discussion}

This study has shown that synovial fluid differs electrically from surrounding tissues. However, impedance varies even inside the synovial capsule indicating that the size of the joint,
Table 3 Confusion matrix of results from different types of needles

\begin{tabular}{lll}
\hline Reality & & \\
\hline Classification & Inside the synovial cavity & Outside the synovial cavity \\
\hline $\begin{array}{l}\text { Synovial fluid detection } \\
\text { 22G BIP needles }\end{array}$ & & \\
Synovial fluid detected & $9(69 \%)$ & $1(7 \%)$ \\
Synovial fluid not detected & $4(31 \%)$ & $14(93 \%)$ \\
Number of injections & 13 & 15 \\
$24 \mathrm{G}$ BIP needles & $50(89 \%)$ & $10(18 \%)$ \\
Synovial fluid detected & $6(11 \%)$ & $47(82 \%)$ \\
Synovial fluid not detected & 56 & 57 \\
Number of injections & &
\end{tabular}


Fig. 2 Example cases of injections. Proportion of detections within a 1-s time interval are shown as a bar plot and the impedance value as the light grey line. Small joints typically provided only Complex detections and large joints provided Pure detections. Needle movements inside the joints affected the measurement results and classification
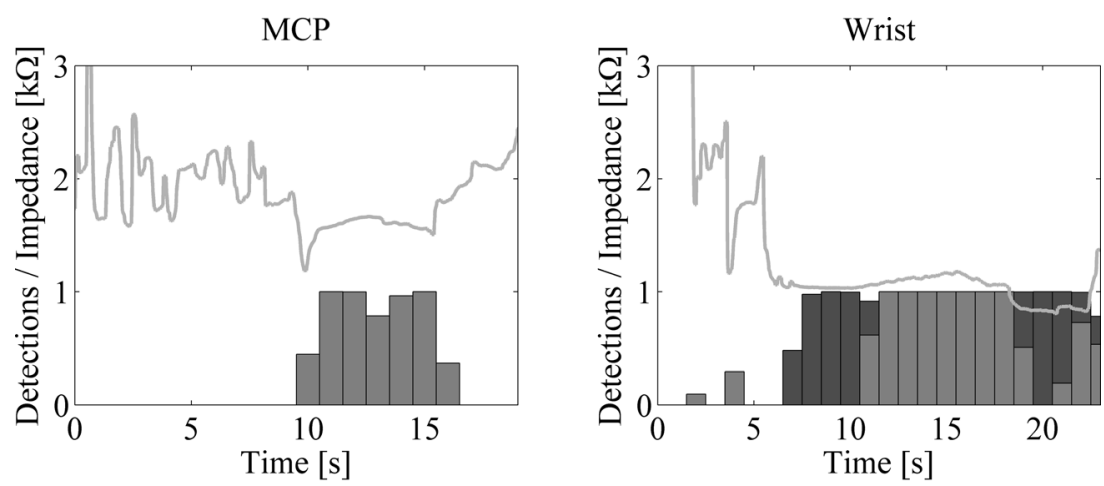

Hip

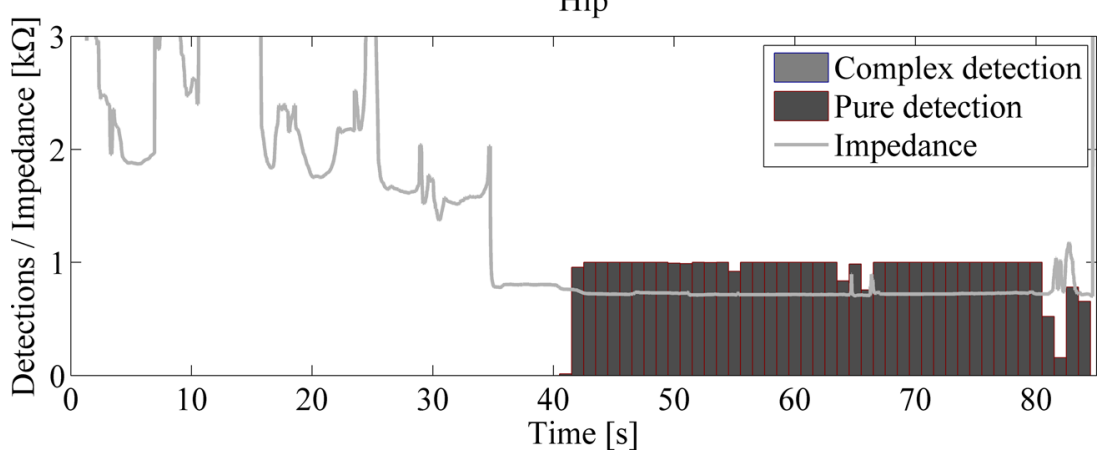

the amount of synovial fluid, the amount of inflammation, and the presence of different abnormalities may affect the bioimpedance properties, challenging the technology.

With $22 \mathrm{G}$ needles, specificity was good at $93 \%$ (68$100 \%$ ), but the sensitivity needs to be improved. In 4 of 13 cases, the device did not detect synovial fluid even though the needle tip is confirmed by ultrasonography to be in the joint cavity. This was probably due to suboptimal classifier parameters. The original model was tuned in the explorative phase with 11 injections, mainly in the knees. It seems likely that the data were too limited for model creation and the model should be updated using all of the new gathered data.

With 24G needles, sensitivity was good at 89\% (78-96\%). False-negative cases were mostly due to software-related sound issues. Careful inspection of the recorded data files revealed that the system had detected synovial fluid at the right time in six cases but no sounds were heard during these events. In addition, the needle tip may have had poor contact

Table 4 Pooled classification results and statistical performance values

\begin{tabular}{ll}
\hline Statistic & Value (\%) and 95\% confidence interval \\
\hline Sensitivity & $85.5(75.0-92.8)$ \\
Specificity & $84.7(74.3-92.1)$ \\
Accuracy & $85.1(78.1-90.5)$ \\
Positive predictive value & $84.3(73.6-91.9)$ \\
Negative predictive value & $85.9(75.6-93.0)$ \\
\hline
\end{tabular}

with well-conducting synovial fluid even inside the joint cavity. There may have been poorly electrically conductive tissue structures inside the joint cavity, such as the articular cartilage surrounding the tip of the bones.

The performance comparison between different needle types or different target joints is also interesting (Tables 2 and 3). However, the number of cases in subgroups was too low, and therefore, reliable comparison cannot be made about the true performance level in subgroups. Statistically, it is relevant to consider only the pooled results. Nevertheless, accuracy was highest in the toes, wrists, and fingers. In 9/9 injections into the metatarsophalangeal (MTP) joints, in 18/ 24 injections into the wrists, and in 9/12 injections into the MCP joints, the device functioned correctly. This is notable, as the small joints are often difficult to inject for inexperienced physicians.

The sensitivity of the system was good, but there were 10 false-positive events out of 58. In four cases, the system incorrectly detected synovial fluid during penetration of the skin or immediately underneath the skin. The duration of these events was very short, usually $0.1 \mathrm{~s}$ or less. Based on the recorded data, it seems that in some cases the bioimpedance of subcutaneous structures strongly resembles that of synovial fluid. This is likely caused by motion artifacts rather than actual subcutaneous structures. As the false detection usually occurs during skin penetration or immediately after it, false detection can be easily disregarded by the operating physician. Thus, in future use, a physician should consider the penetration depth together with the classification result. 
In six cases, the reason for false-positive events was uncertain. Most likely, puncturing even small blood vessels or bursae produces brief but incorrect synovial fluid detections. It is not clear whether reducing the likelihood of these events without altering the sensitivity to real synovial fluid is possible.

The goal of treatment of early RA is remission. The existing treatment strategy has been the active use of sDMARDs with or without GCs. The newer bDMARDs have improved the prognosis of treatment-resistant RA dramatically; however, the cost of these bDMARDs is very high compared with older sDMARDs. In the study by Hørslev-Petersen et al. [16], an aggressive intra-articular injection of triamcinolone (every swollen joint was injected) and sDMARD strategy in early RA provided excellent 2-year clinical and radiological disease control independent of adalimumab induction therapy. Their study showed that good responses can be achieved in a less costly manner by using sDMARDs in combination with intra-articular injections.

The presented novel technology could increase the use of intra-articular injections and as a result decrease the total costs. In high-volume mass production, the prices of tissue-sensing needle can be expected to reach the level of current high-tier styleted needles. The cable is reusable and can be sterilized as needed, and the device can be disinfected; thus, both are intended to long-term use. The bioimpedance analyzer has been designed as a simple and cost-effective device. Overall, the additional costs of the technology are expected to be reasonable. Furthermore, more affordable medication could more than compensate the additional costs of the technology.

The latest analyzer device is suitable for complementary use together with ultrasound. Complementary role could be emphasized in the future by integrating bioimpedance functions to ultrasound imaging devices. Bioimpedance-based needle guidance could also be used independent of ultrasound in health centers to encourage non-specialist physicians to provide intra-articular routine treatment. Furthermore, it could be used in home visits, since the device is so small that it fits into a bag. The device is practical in training, because supervising physician can more easily monitor the puncture procedure. In addition, the device enables recording the procedure and assessing it afterwards.

To conclude, the device provided adequate results and there were no safety issues during this study. Detection of synovial fluid was indicated by an easy method that did not disturb the injection procedure. The performance was good, especially in small joints, which are usually the most difficult for inexperienced physicians. It is also notable that this study was performed specifically in arthritic joints and, therefore, reflects the true clinical picture in practical cases. A better treatment outcome can be achieved when physicians, with the help of BIP Needles, inject actively arthritic joints.
Acknowledgments Injeq Ltd provided BIP Needles and bioimpedance analyzer for the study. We thank all participating patients and study nurses.

\section{Compliance with ethical standards}

Conflict of interests SH, JK, and KK are employees of Injeq Ltd; KK is a stakeholder of Injeq Ltd. Other authors have nothing to declare.

Patient consent Persons gave their informed consent prior to their inclusion in the study.

Ethics approval This clinical study has been approved by the Ethics Committee of the Department of Medicine, Helsinki University Central Hospital, Finland, and has therefore been performed in accordance with the ethical standards laid down in the 1964 Declaration of Helsinki and its later amendments.

\section{References}

1. Möttönen T, Hannonen P, Leirisalo-Repo M, Nissilä M, Kautiainen H, Korpela M et al (1999) Comparison of combination therapy with single-drug therapy in early rheumatoid arthritis: a randomised trial. FIN-RACo trial group. Lancet 353:1568-1573

2. Hollander JL, Brown EM, Jessar RA, Brown CY (1951) Hydrocortisone and cortisone injected into arthritic joints: comparative effects of and use of hydrocortisone as a local antiarthritic agent. JAMA 147(17):1629-1635

3. Courtney P, Doherty M (2013) Joint aspiration and injection and synovial fluid analysis. Best Pract Res Clin Rheumatol 27(2):137-169

4. Taylor WJ, Fransen J, Dalbeth N, Neogi T, Schumacher HR, Brown $M$ et al (2016) Diagnostic arthrocentesis for suspicion of gout is safe and well tolerated. J Rheumatol 43(1):150-153. doi:10.3899/ jrheum. 150684

5. Hetland M, Stengaard-Pedersen K, Junker P, Lottenburger T, Ellingsen T, Andersen LS et al (2006) Combination treatment with methotrexate, cyclosporine and intraarticular betamethasone compared with methotrexate and intraarticular betamethasone in early active rheumatoid arthritis. Arthritis Rheum 54:1401-1409

6. Kuusalo L, Puolakka K, Kautiainen H, Leirisalo-Repo M, Rantalaiho V (2015) Impact of neglecting intra-articular glucocorticoid-injections in remission targeted treatment of early rheumatoid arthritis, EULAR 2015 Congress poster and poster presentation THU0119, Rome

7. Sokka T, Haugenberg G, Asikainen J, Widding HI, Kokko A, Rannio T et al (2012) Similar clinical outcomes in rheumatoid arthritis with more versus less expensive treatment strategies. Observational data from two rheumatology clinics. Clin Exp Rheumatol 31(3):409-414

8. Eriksson JK, Karlsson JA, Bratt J, Petersson IF, van Vollenhoven RF, Ernestam S et al (2015) Cost-effectiveness of infliximab versus conventional combination treatment in methotrexate-refractory early rheumatoid arthritis: 2-year results of the register-enriched randomised controlled SWEFOT trial. Ann Rheum Dis 74:1094-1101

9. Beukelman T, Patkar NM, Saag KG, Tolleson-Rinehart S, Cron RQ, DeWitt EM et al (2011) 2011 American College of Rheumatology recommendations for the treatment of juvenile idiopathic arthritis: initiation and safety monitoring of therapeutic agents for the treatment of arthritis and systemic features. Arthritis Care Res 63(4):465-482

10. Leirisalo-Repo M (2013) What is the best treatment strategy for early RA? Best Pract Res Clin Rheumatol 27:523-536

11. Balint PV, Kane D, Hunter J, McInnes IB, Field M, Sturrock RD (2002) Ultrasound guided versus conventional joint and soft tissue 
fluid aspiration in rheumatology practice: a pilot study. J Rheumatol 29(10):2209-2213

12. Pichler W, Grechenig W, Grechenig S, Anderhuber F, Clement H, Weinberg AM (2008) Frequency of successful intra-articular puncture of finger joints: influence of puncture position and physician experience. Rheumatology (Oxford) 47(10):1503-1505

13. Kalvøy H, Frich L, Grimnes S, Martinsen ØG, Hol PK, Stubhaug A (2009) Impedance-based tissue discrimination for needle guidance. Physiol Meas 30(2):129-140

14. Kari J, Annala K, Annus P, Seppä V-P, Kronström K (2015) A thin needle with bio-impedance measuring probe: tissue recognition performance assessed in in vivo animal study. Available at (http://injeq. com/hallinta/data/files/BRC 3.0, Kari et al. 2015-A thin needle with bio-impedance measuring probe, tissue recognition performance assessed in in vivo animal study.pdf). Accessed 12 May 2015

15. Parmanne P, Kankaanpää E, Mäki S, Kari J, Relas H, Tuompo R et al (2015) Novel injection needle with real-time detection of synovial fluid - exploratory clinical study. EULAR 2015 Congress late breaking abstract and oral presentation, Rome. doi: 10.1136/ annrheumdis-2015-eular.6559

16. Hørslev-Petersen K, Hetland ML, Ørnbjerg LM, Junker P, Pødenphant J, Ellingsen T et al (2015) Clinical and radiographic outcome of a treat-to-target strategy using methotrexate and intraarticular glucocorticoids with or without adalimumab induction: a 2-year investigator-initiated double-blinded, randomized, controlled trial (OPERA). Ann Rheum Dis. doi:10.1136/ annrheumdis-2015-208166 